\title{
Comportamento dos indivíduos e instituições: uma abordagem Vebleniana *
}

\author{
Aline Zulian \\ Solange Regina Marin \\ Orlando Martinelli Júnior ${ }^{* * * *}$
}

\begin{abstract}
Resumo
A Economia tradicional ignora elementos institucionais do comportamento humano. Entretanto, avanços nesta temática incorporam contribuições de outras ciências, como a Psicologia e Sociologia, e resgatam autores que enfatizam a importância das instituições para compreender o comportamento humano nas diferentes sociedades, como é o caso de Thorstein Bunde Veblen. Diferente da concepção de "homem econômico", tradicionalmente entendido como isolado e dotado de racionalidade perfeita, a reflexão deste trabalho está centrada na possibilidade de compreender o comportamento dos indivíduos na Economia com base nas contribuições de Veblen. O objetivo deste artigo é apresentar os aspectos cognitivos e institucionais do comportamento dos indivíduos na Economia, por meio da abordagem vebleniana, que faz referência aos hábitos e aos instintos como forma de abranger as instituições e utiliza a ideia de consumo conspícuo para justificar a presença desses elementos no comportamento. $\mathrm{O}$ estudo do comportamento dos indivíduos considera contextos históricos, interações sociais e instituições.
\end{abstract}

Palavras-chave: Comportamento; Indivíduos; Instituições; Abordagem Vebleniana; Economia Institucional.

\section{Abstract \\ Behaviour of individuals and institutions: a veblenian approach}

Traditional Economics ignores institutional elements of human behavior. However, advances in this subject incorporate contributions from other sciences, such as Psychology and Sociology, to include authors that emphasize the importance of institutions to understand human behavior in different societies, such as Thorstein Bunde Veblen. Unlike the concept of "economic man", traditionally seen as isolated and endowed with perfect rationality, the reflection of this work is focused on the possibility to understand the behavior of individuals in the Economics based on contributions made by Veblen. The purpose of this article is to present the cognitive and institutional aspects of the behavior of individuals in the Economics through the Veblenian approach which refers to the habits and instincts as a way to include the institutions and uses the idea of conspicuous consumption to justify the presence

*Artigo recebido em 16 de maio de 2016 e aprovado em 11 de julho de 2017.

** Doutoranda em Economia do Programa de Pós-Graduação em Economia (PPGE) da Universidade Federal do Rio Grande do Sul (UFRGS), Porto Alegre, RS, Brasil. E-mail: alinezulian@ hotmail.com.

*** Professora do Departamento de Economia e Relações Internacionais e do Programa de Pós-Graduação em Economia (PPGECO) da Universidade Federal de Santa Catarina (UFSC), Florianópolis, SC, Brasil. E-mail: solange.marin@ufsc.br.

${ }^{* * * *}$ Professor do Departamento de Economia e Relações Internacionais e do Programa de Pós-Graduação em Economia e Desenvolvimento (PPGE\&D) da Universidade Federal de Santa Maria (UFSM), Santa Maria, RS, Brasil. E-mail: orlando.martinelli@gmail.com. 
of these elements in behavior. The study of the behavior of individuals considers historical contexts, social interactions and institutions.

Keywords: Behavior; Individuals; Institutions; Veblenian approach; Institutional Economics. JEL B52.

\section{Introdução}

Apesar do estudo sobre indivíduos não ser algo recente na Ciência Econômica, o que se manteve nos tradicionais cursos de Economia foram os pensamentos de cunho neoclássico. Na teoria econômica neoclássica, de acordo com Luz e Fracalanza (2013), o indivíduo é visto como um ser independente e perfeitamente racional, com plenas capacidades cognitivas. Os economistas tradicionais consideram a racionalidade dos indivíduos em um plano axiomático e normativo (Pessali, 2015).

A ideia de "homem econômico" concebe o indivíduo como agente racional e a racionalidade se resume ao cumprimento da premissa de ação racional, resultante de axiomas (Pessali, 2015). Isso transmite a ideia de ser "desnecessária" para um economista a investigação sobre os elementos psicológicos, sociais e institucionais que compõem o comportamento humano.

No entanto, avanços com relação ao estudo dos indivíduos e à maneira como eles se comportam trazem contribuições de outras ciências, como a Psicologia, a Sociologia e a Filosofia, com elementos como a cognição e as instituições, e representam críticas às concepções tradicionais. Não é negado o aspecto racional na espécie humana, mas a racionalidade passa a ter um conceito intersubjetivo, sendo constituída por meio da interação entre as pessoas (Pessali, 2015).

A racionalidade não é isolada e ausente de fatores sociais, ela leva em conta tanto a interação entre os sujeitos quanto a cognição dos indivíduos. De acordo com Nooteboom (2007), esta ideia segue a concepção de uma "cognição incorporada", isto é, apesar de a cognição pertencer ao cérebro e ao corpo do indivíduo, ela também absorve aspectos do meio e da relação entre os indivíduos.

Os aspectos comportamentais da Economia Institucional vebleniana, desde o final do século XIX, trazem elementos de outras ciências para analisar os indivíduos na Economia. Neste sentido, uma das principais contribuições relacionadas aos aspectos institucionais do comportamento dos indivíduos é oferecida por Thorstein Bunde Veblen, considerado um dos precursores do institucionalismo e fundamental para a análise evolucionista da Economia (Hodgson, 2012). Este autor salienta a importância para a Ciência Econômica de orientar-se pelos desenvolvimentos de outras ciências, como a Psicologia e a Sociologia, que estudam o ser humano (Pessali, 2015). 
Veblen (1983) retoma diferentes etapas históricas para descrever as mudanças das condições de emulação de uma sociedade e a predominância de aspectos pecuniários no comportamento. O “consumo conspícuo" de Veblen (1983) compreende o comportamento dos indivíduos com base nos hábitos do pensamento relacionados ao consumo de bens ou serviços que garantam honra, identificados por um sentimento de superioridade em relação às outras pessoas. Para o mesmo autor, o caráter honorífico é encontrado nas pessoas que possuem propriedade desses bens caros e que não estão vinculadas às atividades produtivas. $\mathrm{O}$ indivíduo busca a acumulação de riqueza e almeja a superação do padrão de vida dos demais integrantes do grupo ao qual pertence (“emulação pecuniária”) (Veblen, 1983).

Ao utilizar o "consumo conspícuo", Veblen (1983) descreve como os indivíduos se comportam conforme as instituições e o contexto histórico de uma sociedade. A abordagem vebleniana considera as interações entre os indivíduos, a cognição, as instituições e o meio social, o que diferencia os trabalhos de Veblen dos tradicionais em Economia e reforça o aspecto ontológico desta ciência (Conceição, 2012).

Para compreender o comportamento dos indivíduos, Veblen (1914) utiliza os hábitos e os instintos. Estes aspectos institucionais possuem características cognitivas, uma vez que também envolvem processos do pensamento (Hodgson, 1993). Os hábitos são formações sociais e os instintos possuem, para Veblen (1914), uma característica peculiar, que vai além do conceito biológico da natureza humana, pois incluem a teleologia. Assim, é preciso problematizar a ideia de "homem econômico" que, na concepção de Luz e Fracalanza (2013), é um ser divinamente individualizado, independente, cognitivamente perfeito, imune a qualquer influência social e histórica.

Da mesma forma que as habilidades cognitivas não são isoladas e geradas apenas pela fisiologia humana, os indivíduos se constituem, em relações interindividuais, como seres sociais, de modo que é fundamental considerar os aspectos institucionais do comportamento (Hodgson, 1998). Então, diferente da concepção de "homem econômico", que tradicionalmente é entendido como isolado, desprovido de dificuldades cognitivas e dotado de racionalidade perfeita, o problema deste artigo está centrado na possibilidade de compreender o comportamento dos indivíduos na Economia com base nas contribuições de Veblen.

Para atender o objetivo deste artigo, que é apresentar os aspectos cognitivos e institucionais do comportamento dos indivíduos na Economia, por meio da abordagem vebleniana, serão desenvolvidas três seções, além desta introdução. Inicialmente, são expostas algumas contribuições de Veblen sobre instituições e comportamento, com ênfase na ideia de consumo conspícuo como forma de contextualizar a existência de hábitos e instintos em uma sociedade. Em um segundo 
momento, é feita a caracterização vebleniana dos "hábitos" e, em seguida, são apresentados os "instintos" na abordagem de Veblen. Por fim, fazem-se algumas considerações.

\section{Instituições e comportamento em Veblen}

Thorstein Bunde Veblen, economista e sociólogo americano, é um dos principais pilares da Economia Institucional com ênfase nas discussões sobre instituições e comportamento dos indivíduos (Cosgel, 1997; Hodgson, 2012). De acordo com Hodgson (1998), Veblen possui influência evolucionária e multidisciplinar, e foi o primeiro economista que elaborou uma teoria econômica e institucional com bases darwinianas.

Veblen pertence ao grupo dos chamados "velhos" institucionalistas ${ }^{1}$ e entende que o indivíduo não deve ser considerado isolado e dado por natureza, mas como pertencente a uma sociedade que interage com as instituições (Hodgson, 1998). A abordagem vebleniana reforça a importância de a Ciência Econômica utilizar contribuições da Psicologia, da Sociologia e da Antropologia ${ }^{2}$ para compreender o comportamento humano e estudar elementos que antecedem a ação, que é o caso dos assim chamados hábitos e instintos (Pessali, 2015).

Veblen (1919) apresenta dois fundamentos básicos: a "razão suficiente" e a "causa eficiente". O primeiro, de forma geral, se refere aos agentes racionais e inteligentes, que se baseiam em eventos futuros para se comportarem no presente, sendo a ação econômica vista como teleológica. A "causa eficiente" considera, por outro lado, relações processuais de causa e efeito, mudanças das variáveis com o passar do tempo e estímulos dos hábitos e convenções (Veblen, 1919).

Este entendimento, baseado na "causa eficiente", é considerado por Veblen (1919) como uma "visão moderna" sobre o estudo do comportamento humano.

The modern scheme of knowledge, on the whole, rests, for its definitive ground, on the relation of cause and effect; the relation of sufficient reason being admitted only provisionally and as a proximate factor in the analysis, always with the unambiguous reservation that the analysis must ultimately come to rest in terms of cause and effect (Veblen, 1919, p. 238).

(1) Outros institucionalistas que pertencem ao grupo da "velha" economia institucional são Wesley Mitchell, John R. Commons e Clarence Ayres (Rutherford, 1994).

(2) Thorstein Veblen utiliza elementos da Antropologia, da Sociologia e da Psicologia para compreender o comportamento humano na Economia. Autores como Herbert Simon, George Katona, Tibor Scitovsky e, mais recentemente, Richard Thaler, Daniel Kahneman e Amos Tversky trazem aproximações da Economia com a Psicologia. Recentemente, Geoffrey. Hodgson e John B. Davis são exemplos de autores que aproximam a Economia de elementos da Filosofia, Sociologia e da Psicologia. 
Isto vai ao encontro do que é chamado de "causação cumulativa", em que Veblen (1998) destaca que a Economia deveria ser uma ciência evolucionária, com uma análise processual, não teleológica e não taxonômica. Nas palavras de Veblen (1909, p. 628), o crescimento da cultura é, por exemplo, causado por um processo cumulativo de habituação, de modo que este caráter cumulativo ocorre "[...] because each new situation is a variation of what has gone before it and embodies as causal factors all that has been effected by what went before".

Conforme Conceição (2002, p. 96), este entendimento de processo se refere ao fato de que "[...] o desenvolvimento da atividade econômica não pode ser entendido como algo preestabelecido, esperado, ou resultado 'ideal' da convergência ao equilíbrio". Na verdade, de acordo com o mesmo autor, o que ocorre é um processo histórico de mudança que pode ser positivo (avanço) ou não (retrocesso), uma vez que, para Veblen (1998), evolução é diferente de uma melhora em uma situação.

Apesar de Veblen (1919) não desconsiderar todos os aspectos da "razão suficiente", para ele, as instituições se relacionam com o fenômeno da "causa eficiente", pois introduzem os hábitos e as preferências dos indivíduos. "The wants and desires, the end and aim, the ways and means, the amplitude and drift of the individual's conduct are functions of an institutional variable that is of a highly complex and wholly unstable character" (Veblen, 1919, p. 242-243).

O estudo do comportamento dos indivíduos, em uma abordagem institucionalista vebleniana, leva em conta tanto os aspectos da "causa eficiente" quanto da "razão suficiente", apesar de estar focado no primeiro. Os hábitos são essenciais na obra de Veblen (1919), são parte da investigação sobre o surgimento das instituições, e envolvem as formações sociais e materiais de uma sociedade. As instituições são os hábitos de pensamento de uma sociedade, isto é, uma instituição "[...] is a complex of habits of thought which reflect the habits of life embodied in the institutional structure of society" (Veblen, 1919, p. 44).

Uma maneira dos hábitos de vida surgirem em uma sociedade é via exigências materiais. Veblen (1914) chama essas exigências criadas socialmente de "the state of the industrial arts", uma vez que elas impõem certos padrões de comportamento na sociedade, sendo constituídas por heranças culturais.

The state of the industrial arts is a fact of group life, not of individual or private initiative or innovation. It is an affair of the collectivity, not a creative achievement of individuals working self-sufficiently in severalty or in isolation. In the main, the state of the industrial arts is always a heritage out of the past; it is always in process of change, perhaps, but the substantial body of it is knowledge that has come down from earlier generations (Veblen, 1914, p. 103). 
Os hábitos de vida, motivados principalmente por essas mudanças materiais, provocam os hábitos de pensamento, que são formados por fenômenos imateriais (Silva, 2010). Ao considerar que a sociedade cria certas exigências ou padrões de vida e de pensamento a serem seguidos por todos, e que são passados de uma geração para outra, Veblen (1914) entende o comportamento do indivíduo com base em aspectos institucionais. Ou seja, o comportamento do "homem vebleniano"3 é complexo e se compõe não apenas de hábitos, mas também de instintos (Hodgson, 1998).

Antes de caracterizar o comportamento humano com base nos instintos e nos hábitos, Veblen (1983) propõe uma contextualização por meio da discussão sobre o "consumo conspícuo", que traz os hábitos e os instintos de uma sociedade, em especial da "classe ociosa", em diferentes momentos históricos. Apesar de existirem autores que tratam o "consumo conspícuo" com foco na atividade de consumo, percebe-se que Veblen destina sua atenção para os processos que envolvem o comportamento em um sentido geral; o consumo é apenas uma maneira de demonstrar as manifestações pecuniárias e culturais que, por meio dos instintos e hábitos, compõem os indivíduos ${ }^{4}$.

Na obra "A Teoria da Classe Ociosa: Um Estudo Econômico das Instituições”, publicada em 1899, Veblen retoma inicialmente aspectos históricos e institucionais que compõem o comportamento dos indivíduos (Veblen, 1983). A chamada "classe ociosa" surge na passagem da selvageria primitiva para o estágio de barbárie, quando é instituída a propriedade. Este segmento social é visto como ligado às atividades "dignas", que são desvinculadas do aspecto produtivo das atividades industriais e rotineiras ${ }^{5}$ (Veblen, 1983).

A passagem de uma fase histórica para outra pode indicar mudanças nos hábitos de vida, como ocorreu na fase inicial da sociedade humana, em que a luta era considerada como algo honorífico (Veblen, 1983). No entanto,

[...] o critério na classificação dos fatos muda à medida que a cultura evolui, isso porque muda o fim, em função do qual se apreendem tais fatos, mudando o ponto de vista dominante. Em suma, os traços salientes e decisivos de um tipo de atividade ou de uma classe social num determinado estágio de cultura não terão a mesma importância relativa para os fins de classificação num estágio subsequente (Veblen, 1983, p. 8).

(3) Sobre a interpretação da crítica de Veblen ao "homem econômico" ver Hodgson (1993).

(4) Alguns autores utilizam o termo "instituições de consumo" para tratar da discussão que envolve o comportamento de consumo e as instituições, como é o caso de Cosgel (1997) e Dolfsma (2002). Além disso, esta discussão pode se direcionar para o consumo como objeto central de análise, caso de Dalal (2014).

(5) As pessoas que participavam de atividades como esporte, sacerdócio, exército, ou seja, de funções resultantes do ócio e da emulação, eram consideradas nobres e pertencentes a "classe ociosa"; enquanto os indivíduos ligados ao trabalho industrial eram vistos como "indignos" (Veblen, 1983). 
A mudança de padrões de comportamento ocorre lentamente e "assim que a tradição se firma, o senso comum da comunidade a erige em regra de conduta [...]" (Veblen, 1983, p. 10). O aspecto normativo das instituições está na formação social de hábitos de pensamento, como é o caso da crença, identificada por Veblen (1983), de que o trabalho industrial não possui prestígio e, por isso, é desligado da classe social superior.

Com o passar do tempo, o padrão de vida exigido por uma sociedade pode se modificar, como é o caso dos bens de consumo de caráter honorífico. De acordo com Dalal (2014), antes da Segunda Guerra Mundial, as pessoas que possuíam carros eram vistas como privilegiadas e superiores; mas, com o passar dos anos e com a generalização deste hábito, a posse de automóveis perdeu o status de riqueza, a não ser para carros de luxo.

Uma vez inseridas na sociedade, as instituições podem se modificar de forma gradual, positiva ou negativamente. Exemplo disso é, para Veblen (1983), a deterioração dos hábitos de boas maneiras com o avanço industrial.

[...] As maneiras e o comportamento dos homens, nas modernas comunidades industriais, mesmo no seio das classes mais altas, são infelizmente ruins, a decadência do código de bom tom, nas classes especificamente industriais, isto é, a vulgarização da vida, se tornou uma das principais enormidades da civilização de nossos dias, aos olhos de todos que têm sensibilidade delicada. [...] O decoro é produto e sintonia da existência de uma classe ociosa e que somente se desenvolve plenamente num regime de status (Veblen, 1983, p. 2526).

Alguns hábitos que pertencem apenas às classes superiores podem, no decorrer do tempo, difundir-se a fim de tornarem-se uma utilidade intrínseca, ou uma instituição. Conforme Veblen (1983), os hábitos podem permanecer ou passar por mudanças, e as condições de emulação tendem a se alterar. Na fase primitiva, as condições de emulação estavam vinculadas ao instinto pecuniário que dependia do hábito de pensar que medalhas e premiações resultantes de lutas garantiriam a superioridade. Com a evolução da sociedade, de acordo com o mesmo autor, esse caráter honorífico passa também a ser concedido para a posse de bens de consumo e serviços, de modo que um dos motivos para almejar a propriedade ${ }^{6}$ é exatamente a emulação. Com a evolução da indústria, as pessoas passaram a demandar bens muito além do necessário para a subsistência, o que iniciou uma "competição pelo aumento dos confortos da vida, principalmente pelo aumento dos confortos físicos que o consumo de bens proporciona" (Veblen, 1983, p. 16).

Com o avanço da indústria, a riqueza passa a ser transmitida visualmente aos demais por meio da aquisição de bens e de serviços sofisticados. Ter riqueza não é

(6) A classe ociosa e a propriedade são duas instituições que surgiram juntas (Veblen, 1983). 
suficiente para conferir admiração, é preciso que o poder seja transmitido e visível para os demais. Esta disputa por egos, segundo Veblen (1983), leva à definição de "emulação pecuniária", que é justamente a busca por possuir um padrão de riqueza visível e que esteja acima dos demais indivíduos. Para o autor, não há um limite ou um padrão ótimo de emulação; o desejo de riqueza e a demonstração desta nunca se exalam.

Veblen (1983) inclui no princípio do consumo conspícuo, composto de normas que tornam o gasto honorífico, a existência de hábitos de pensamento sobre o senso da beleza, da obrigação e da verdade científica. As pessoas não se comportam de maneira isolada, são movidas também por grupos ou classes sociais, que incentivam o sentimento de poder pecuniário. Para Veblen (1983), na "lei seletiva do consumo conspícuo" existem regras de consumo para fazer com que o indivíduo alcance certo nível de gastos, sendo que essas normas se transformam em hábitos de pensamento. Isto demonstra que os interesses econômicos não estão isolados, relacionam-se com os hábitos e com os instintos de uma sociedade.

Para o estudo do comportamento, Veblen (1983) considera fundamental tratar de instituições e, para chegar nisso, é necessário passar pelos instintos e hábitos, o que de certa maneira vincula aspectos institucionais e cognitivos do comportamento humano. Conforme Pessali (2015), desde Veblen, economistas não tradicionais reforçam a importância de considerar elementos de outras áreas para tratar de comportamento humano, como é o caso da psicologia cognitiva. "A cognição nos leva a pensar e agir de certa maneira - o que chamamos de comportamento. O comportamento, por sua vez, expõe em grande parte nossos processos cognitivos, possibilitando que sejam regularmente revisados" (Pessali, 2015, p. 51). O hábito, um dos elementos institucionais citados por Veblen, está relacionado à cognição que, segundo Pessali (2015), refere-se a uma propensão em repetir uma ação ou um pensamento.

\section{Instituições e comportamento: os hábitos veblenianos}

Veblen (1983), ao estudar o comportamento humano, considera os processos anteriores à ação, os quais incluem os hábitos e os instintos. Como há limitações de pensamento, os indivíduos baseiam-se em estímulos habituais existentes. Desta forma, considerar o comportamento em um contexto processual é passar por aspectos que envolvem o contexto histórico do ambiente, os hábitos de uma sociedade e os instintos humanos.

Os hábitos são resultados de fatores passados, e compõem o comportamento dos indivíduos tanto no ambiente de trabalho quanto no de lazer. Conforme Veblen (1914), o surgimento da indústria impõe mudanças nas atividades habituais relacionadas à mão de obra. A força muscular é substituída por processos mecânicos, 
de modo que esta mudança afeta principalmente as classes de trabalhadores envolvidas com os trabalhos manuais da indústria. Com o passar do tempo, isso também interfere nos hábitos da vida e da mente dos indivíduos e, consequentemente, em seus comportamentos ${ }^{7}$ (Veblen, 1914).

Por mais que os hábitos sejam fundamentais na análise vebleniana do comportamento, de acordo com Hodgson (2002), é preciso destacar que estes não equivalem a mesma definição de comportamento, pois são uma propensão. Conforme esse autor, os hábitos não são vistos como resultantes de um comportamento, mas como geradores, estão ligados ao conhecimento e às crenças. Como no caso da substituição da força humana pela força mecânica, os comportamentos e os pensamentos podem resultar de mudanças nas circunstâncias do meio e nos hábitos de uma sociedade. "All ideas, including beliefs, preferences, and rational modes of calculation, were regarded as evolutionary adaptations to circumstances, established through the acquisition of habitual propensities" (Hodgson, 1998, p. 179).

Os hábitos não exigem grandes esforços da mente, isto é, estão relacionados com costumes familiares e atividades rotineiras já existentes (Veblen, 1914). Os indivíduos não possuem dificuldades em realizar funções que estão habituados a fazer. Conforme Hodgson (1988), quando um indivíduo compra um carro, pode inicialmente pensar em quais momentos específicos irá usá-lo. Mas, após um tempo frequente de uso, andar de carro, ao invés de usar o ônibus ou andar a pé, pode ter se tornado um hábito, sem fazer grandes esforços da mente. "Whatever their origin, repeated acts tend to congeal into habits, and become removed from the sphere of rational deliberation in the mind" (Hodgson, 1988, p. 127).

Mesmo que possa existir a perpetuação de um hábito, a transmissão deste entre as gerações pode sofrer alterações com o tempo, uma vez que se adquire conhecimento em diferentes etapas históricas (Veblen, 1914).

What men can do easily is what they do habitually, and this decides what they can think and know easily. They feel at home in the range of ideas which is familiar through their everyday line of action. A habitual line of action constitutes a habitual line of thought, and gives the point of view from which facts and events are apprehended and reduced to a body of knowledge. What is consistent with the habitual course of action is consistent with the habitual line of thought, and gives the definitive ground of knowledge as well as the conventional standard of complacency or approval in any community.

(7) De acordo com Monasterio (1998), a definição aplicada às instituições aproxima-se do contexto vivido na época de Veblen, em que de um lado estava a manufatura ainda de caráter teleológico e do outro estava a indústria, em uma análise processual. Para esse mesmo autor, um hábito, seguido por um grupo de pessoas, institucionalizase e influencia a criação de outros comportamentos, podendo gerar novos hábitos, outras instituições, e assim por diante. 
Conversely, a processor method of life, once understood, assimilated in thought works into the scheme of life and becomes a norm of conduct, simply because the thinking, knowing agent is also the acting agent. What is apprehended with facility and is consistent with the process of life and knowledge is thereby apprehended as right and good (Veblen, 1898, p. 195).

Conforme Veblen (1898), quando um fator interfere nos hábitos de vida de uma sociedade, uma vez compreendido e difundido entre as pessoas, interfere também nos hábitos de pensamento e torna-se uma norma de comportamento. $\mathrm{O}$ comportamento dos indivíduos não é algo isolado, está ligado aos instintos e aos hábitos de pensamento, o que forma certos padrões na vida em uma sociedade. "The individual is but a single agent in each case; and he enters into each successive action as a whole, although the specific end sought in a given action may be sought avowedly on the basis of a particular interest" (Veblen, 1998, p. 412).

O período histórico que compreende a análise das instituições e do comportamento dos indivíduos também precisa ser considerado. A história das relações econômicas de uma sociedade implica a história de vida desta, uma vez que os interesses econômicos se relacionam à estrutura cultural (Veblen, 1998). As exigências históricas de sobrevivência e de manutenção da vida humana podem formar novos hábitos de vida e de pensamento. "Changes in the material facts breed further change only through the human factor" (Veblen, 1998, p. 410).

Essa interação da história com os instintos e com os hábitos permite uma análise da sociedade pautada não apenas em elementos materiais, mas também inclui as formas de pensar de cada indivíduo. “[...] A própria percepção do instinto em Veblen é aprumada pelos fatores imateriais que balizam a ação social. Tais fatores, definidos como hábitos de pensamento, corrigem, de certo modo, por meios sociais, as propensões instintivas de natureza inata" (Silva, 2010, p. 298).

É por isso que na concepção vebleniana é preciso considerar instintos e hábitos conjuntamente e não ignorar o período histórico da análise. As características habituais também podem gerar padrões de vida em uma sociedade. "O padrão de vida é pela sua natureza um hábito. [...]. A dificuldade em abandonar um padrão estabelecido consiste na dificuldade em quebrar um hábito já formado" (Veblen, 1983, p. 51). Mesmo que existam pressões por mudanças, os indivíduos são resistentes às alterações nos seus padrões de vida, uma vez que os hábitos antigos já estão fortemente enraizados na cultura.

Em Veblen, a instituição pode ser vista como resultado de um comportamento padrão que foi seguido por um grupo de pessoas no decorrer de um tempo, como é o caso dos hábitos culturais. Um conjunto cumulativo de hábitos constitui a cultura de uma sociedade e uma consequência desses hábitos de pensamento difundidos é a formação de instituições (Veblen, 1919). 
As instituições são elas próprias o resultado de um processo seletivo e adaptativo que modela os tipos prevalecentes, ou dominantes, de atitudes e aptidões espirituais; são, ao mesmo tempo, métodos especiais de vida e de relações humanas, e constituem, por sua vez, fatores eficientes de seleção (Veblen, 1983, p. 87).

Um hábito antigo é mais difícil de ser esquecido ou substituído por outro. "Em geral, quanto mais antigo o hábito, tanto mais inquebrantável; e quanto mais um determinado hábito coincidir com os costumes, tanto maior a persistência com que se fixará" (Veblen, 1983, p. 51). A mente humana se direciona para os hábitos passados que resistiram por diferentes gerações ao longo do tempo, constituindo o comportamento dos indivíduos.

Conforme Veblen (1914), ao mesmo tempo em que o hábito é transmitido entre gerações por meio da educação, tradição e formação, ele também cria costumes e convenções. Assim como um conjunto de hábitos de pensamento se difunde e se configura como instituições que moldarão o comportamento, as instituições podem restringir comportamentos e desenvolver novos hábitos. Elas estão presentes nas decisões dos indivíduos e são, em alguma medida, a "materialização de modelos mentais compartilhados que vemos operar na forma de convenções sociais, regras de conduta, códigos legais e organizações sociais de naturezas diversas - incluindo as com propósitos primordialmente econômicos" (Pessali, 2015, p. 129-130).

Diferente de considerar o indivíduo isolado e a gênese das instituições individualismo metodológico -, é preciso atentar para os "modelos mentais" compartilhados entre as gerações, que incluem hábitos, valores, regras e outras instituições (Pessali, 2015). Mesmo que o indivíduo seja único, detentor da capacidade de decisão final, a formação do comportamento sofre interferências do fator histórico, social e institucional. Ocorrem mudanças na estrutura social desde que existam alterações conjuntamente nos hábitos do pensamento (instituições), ou seja, "a evolução da sociedade é substancialmente um processo de adaptação mental de parte do indivíduo [...]" (Veblen, 1983, p. 88).

A situação de hoje modela as instituições de amanhã mediante um processo seletivo e coercitivo, atuando na habitual opinião humana sobre as coisas, e assim alterando, ou envigorando um ponto de vista ou uma atitude mental herdada do passado. As instituições - o que vale dizer, os hábitos mentais sob a orientação das quais os homens vivem são, por assim dizer, herdadas de uma época anterior; época mais ou menos remota, mas, em qualquer caso, elaboradas no passado e dele herdadas. As instituições são o produto de processos passados, adaptados a circunstâncias passadas, e por conseguinte nunca estão de pleno acordo com as exigências do presente (Veblen, 1983, p. 88). 
O aparato institucional que hoje vigora é, segundo Veblen (1983), resultado de padrões seguidos no passado e pode ter sofrido alterações motivadas principalmente por forças econômicas. Conforme o mesmo autor, as instituições econômicas incluem "métodos habituais de dar continuação ao modo de vida da comunidade em contato com o ambiente material no qual ela vive", e permitem, por um lado, o seu conservadorismo, mas, por outro lado, estão sujeitas às pressões externas por mudanças, que não ocorrem apenas no ambiente, mas também no próprio indivíduo. Quando ocorrem alterações de ordem econômica, isso também implica em mudanças nos hábitos e nos comportamentos dos indivíduos. "What is true of the individual in this respect is true of the group in which he lives. All economic change is a change in the economic community, - a change in the community's methods of turning material things to account" (Veblen, 1998, p. 412).

Por outro lado, além das forças que pressionam por mudanças, alguns hábitos podem se manter por um longo tempo em uma sociedade, pois alterá-los implica maior esforço da mente na direção de um novo padrão, sendo este um dos motivos que explica o conservadorismo das classes e das instituições (Veblen, 1983).

Essas instituições assim herdadas, esses hábitos mentais, pontos de vista, atitudes e aptidões mentais, ou seja lá o que for, são, portanto, um elemento conservador; e esse é um fator de inércia social, de inércia psicológica, de conservantismo (Veblen, 1983, p. 88).

Veblen (1983) chama de "conservantismo" uma das características presentes na classe social superior, uma vez que possui hábitos mais fortemente enraizados e não cede facilmente às mudanças. $\mathrm{O}$ grupo a que o indivíduo pertence possui um padrão de vida com os sinônimos do que é certo e bom; uma mudança neste padrão, caso ocorra, acontece lentamente e é motivada principalmente por forças econômicas ("pressão pecuniária") (Veblen, 1983). Isto também se deve a uma característica cognitiva dos indivíduos: a aversão ou o repúdio às mudanças, que foi retratada por Veblen e também é sinalizada atualmente pela economia comportamental.

A aversão à mudança é em grande parte uma aversão ao incômodo de precisar fazer o reajustamento exigido por qualquer determinada mudança; e essa solidariedade ao sistema de instituições de qualquer determinada cultura de um povo fortalece a resistência instintiva oposta a qualquer mudança nos hábitos mentais humanos, até mesmo em assuntos que, em si mesmos, não têm maior importância (Veblen, 1983, p. 93).

Para Veblen (1983), não são tanto as alterações nos hábitos de pensamento que são desagradáveis, mas o fato de que as mudanças envolvem certo grau de esforço da mente e exigem grande nível de energia pessoal. Uma vez que uma instituição é inserida e aceita como padrão de vida por um grupo de indivíduos, estes terão seus hábitos de pensamento convergentes e passarão a vigiar o comportamento dos demais indivíduos, para que não ocorram desvios de conduta. 
O que ocorre, para Veblen (1983), é que os indivíduos estão inseridos em um processo de seleção com base nos comportamentos que seguirão. Quando um padrão de vida é tido como melhor ou ideal, passa a ser perseguido pelas pessoas e, quem não o faz, é excluído pelo grupo a que pertence ou a que gostaria de pertencer.

Logo que determinada propensão ou determinado ponto de vista foram aceitos como padrão ou norma de vida autorizados, estes reagirão no caráter dos membros da sociedade que os aceitaram, e até certo ponto, modelarão seus hábitos mentais e exercerão uma vigilância seletiva no desenvolvimento das aptidões e das inclinações do homem. [...]. O material humano que não se preste aos métodos de vida impostos pelo esquema adotado sofre uma tal ou qual eliminação, bem como uma repressão (Veblen, 1983, p. 97).

O indivíduo possui habilidades cognitivas e hábitos de pensamento, além de existir o instinto que está presente antes mesmo do conjunto de hábitos formados ao longo da vida. Esse caráter habitual concede certas regularidades de comportamentos. Neste sentido, "the strong influence of institutions upon individual cognition provides some significant stability in socioeconomic systems, partly by buffering and constraining the diverse and variable actions of many agents" (Hodgson, 1998, p. 171). Não apenas os hábitos envolvem processos do pensamento e são essenciais para o estudo do comportamento humano, é preciso considerar também os instintos veblenianos.

\section{Instituições e comportamento: os instintos veblenianos}

Os instintos ganham espaço especial na análise de Veblen (1914), pois são os "principais motores" do comportamento humano, que admite a dificuldade de apresentar um conceito exato e geral para o instinto, e afirma que a sua proposta também não é essa. Mesmo assim, a incerteza na definição de instintos não implica que o termo não deve ser utilizado, mas que, na verdade, pode envolver diferentes concepções (Veblen, 1914).

Enquanto em Hodgson (2010) o conceito de instinto vebleniano mantém um caráter biológico ${ }^{8}$ - representação de algo inato à natureza humana -, outros intérpretes consideram ser este apenas um ponto de partida. A direção dada por Veblen (1914) para a delimitação dos instintos não segue somente uma natureza fisiológica, ou tropismática ${ }^{9}$, e nem exclusivamente um âmbito consciente. Os

(8) "Ordinariamente as ciências da natureza tratam do instinto como resposta automática dos seres vivos, sob determinadas condições, às demandas do meio e àquelas provocadas por outros seres vivos. Contudo, a definição biológica de instinto não está muito próxima daquela pretendida por Veblen” (Silva, 2010, p. 291).

(9) Os tropismos são características apenas fisiológicas, individuais, sem qualquer interferência social ou institucional (Veblen, 1914). Para Veblen (1914, p. 9), "the recognised tropisms stand out, to all appearance, as sharply defined physiological traits, transmissible by inheritance intact and unmodified, separable and unblended, in a manner suggestively like the unit characters spoken of in latter day theories of heredity". 
instintos possuem características de ambas as dimensões e o que os diferencia é o objetivo final a ser alcançado. Este propósito último a ser atingido pelo instinto, que precisa ser pensado, caracteriza-o como teleológico e consciente (Veblen, 1914).

Instinctive action is teleological, consciously so, and the teleological scope and aim of each instinctive propensity differs characteristically from all the rest. The several instincts are teleological categories, and are, in colloquial usage, distinguished and classed on the ground of their teleological content. As the term is here used, therefore, and indeed as it is currently understood, the instincts are to be defined or described neither in mechanical terms of those anatomical or physiological aptitudes that causally underlie them or that come into action in the functioning of any given instinct, nor in terms of the movements of orientation or taxis involved in the functioning of each. The distinctive feature by the mark of which any given instinct is identified is to be found in the particular character of the purpose to which it drives. "Instinct", as contra-distinguished from tropismatic action, involves consciousness and adaptation to an end aimed at (Veblen, 1914, p. 3-4).

É importante deixar claro que a característica teleológica do instinto vebleniano possui um aspecto peculiar. O caráter teleológico do ato instintivo, de acordo com Veblen (1914), está no seu propósito, no seu objetivo de atingir um fim. No entanto, para consegui-lo, exige-se certa inteligência, variando em grau conforme o instinto que está sendo analisado. Por mais que o instinto possua um aspecto teleológico (direciona-se para um fim específico), envolve os processos da mente, de modo que a ação humana, no sentido geral, não pode ser entendida como teleológica, uma vez que o comportamento, que irá resultar em uma decisão, envolve diferentes aspectos cognitivos e institucionais. O instinto envolve tanto a fisiologia do indivíduo quanto a sua capacidade de deliberar.

The ends of life, then, the purposes to be achieved, are assigned by man's instinctive proclivities; but the ways and means of accomplishing those things which the instinctive proclivities so make worthwhile are a matter of intelligence. It is a distinctive mark of mankind that the working-out of the instinctive proclivities of the race is guided by intelligence to a degree not approached by the other animals (Veblen, 1914, p. 5-6).

Ao mesmo tempo em que Veblen (1914) traz os instintos para a discussão institucional e social, algo que é tradicionalmente visto como uma característica biológica do ser e pertencente ao inconsciente, o autor também direciona seu conceito para a racionalidade e para a inteligência dos indivíduos. O instinto pode ser aparentemente simples, mas envolve os diversos meios e formas para atingir o fim desejado, passa por hábitos de pensar e agir. Compreender os instintos tanto de um ponto de vista racional e teleológico, com o alcance de um fim específico por meio da inteligência humana, quanto do aspecto inconsciente, possibilita um 
distanciamento do pressuposto da racionalidade perfeita ${ }^{10}$, que é tradicionalmente considerado na Economia.

Não há como desvincular os instintos, os hábitos e a razão. Conforme Hodgson (2010), a linguagem, por exemplo, apesar de ser impossível de ser desenvolvida sem uma interação social e sem o uso do raciocínio, também não é possibilitada sem instintos primários. Para o mesmo autor, é inegável a importância do hábito e das formações sociais que compõem o indivíduo no processo de interação com a sociedade e, ao mesmo tempo, é fundamental a compreensão do comportamento com base nos instintos.

Both instinct and habit are vital for individual development. Inherited dispositions are necessary for socialization to begin its work. Obversely, much instinct cannot manifest itself without the help of culture and socialization. Instinctive behaviour and socialization are not always rivals but often complements: they interact with one another. The degree to which we are affected by our social circumstances is immense, but that is no ground for the banishment of the concept of instinct from social theory (Hodgson, 2010, p. 3).

É por isso que, em um primeiro momento, quando Veblen (1914) analisa separadamente os instintos em uma dimensão fisiológica e em uma dimensão consciente, parece haver a necessidade de delimitar qual possui maior importância analítica. No entanto, esta é uma tarefa difícil, e o próprio Veblen não identifica uma relação primordial e causal entre as dimensões.

Os hábitos de uma sociedade, os instintos - em diferentes graus de consciência - e a história caminham juntos. Em outras palavras, além de os instintos veblenianos constituírem fatores fisiológicos e racionais, possuem características constituídas pelo contexto histórico e social. Apesar de ser difícil classificar os instintos, Veblen (1914) aponta na direção de alguns "instintos universais", que podem ser divididos em: "inclinação paternal", "curiosidade vã”, "instinto de trabalho eficiente" ou "instinto para artesanato" e "instinto predatório" (Monasterio, 1998).

O instinto de "inclinação paternal" inclui, segundo Veblen (1914), a preocupação dos indivíduos com a sobrevivência e com o bem-estar de seu grupo familiar. Este instinto representa muito mais do que a procriação e a manutenção do bem-estar dos filhos, envolve a preocupação com a vida das próximas gerações e caracteriza um sentimento de preservação e garantia dos descendentes.

So also, virtually all thoughtful persons, - that is to say all persons who hold an opinion in these premises, - will agree that it is a despicably inhuman thing for the current generation willfully to make the way of life harder for the next

(10) Pessali (2015) traz a ideia de "racionalidade intersubjetiva", constituída por meio da interação entre as pessoas. 
generation, whether through neglect of due provision for their subsistence and proper training or through wasting their heritage of resources and opportunity by improvident greed and indolence. Providence is a virtue only so far as its aim is provision for posterity (Veblen, 1914, p. 26).

O instinto de "inclinação paternal" visa garantir a sobrevivência das gerações posteriores, o que seria o caso, por exemplo, da preservação do meio ambiente e da administração dos recursos financeiros para que os descendentes não sejam prejudicados (Veblen, 1914).

O instinto da "curiosidade vã" está no fato de os indivíduos buscarem "conhecimento pelo conhecimento", de modo a tratar-se de algo "vão", sem relação monetária direta ${ }^{11}$ (Monasterio, 1998). Desta forma, o conhecimento relacionado ao fenômeno da "curiosidade vã" é algo característico do ser humano; o indivíduo compreende a realidade sem necessariamente utilizar conhecimentos aplicados e aprendidos ao longo do tempo. Essa curiosidade é um conhecimento circunstancial e sem a preocupação e a necessidade de ser adquirido.

Human curiosity is doubtless an idle propensity, in the sense that no utilitarian aim enters in its habitual exercise; but the material information which is by this means drawn into the agent's available knowledge may none the less come to serve the ends of workmanship (Veblen, 1914, p. 88).

Além destes, há o "instinto de trabalho eficiente" e o "instinto predatório"12. O ser humano é considerado, conforme Veblen (1898), um "animal social e pacífico", que possui como um de seus principais motivadores para um trabalho bem feito, o "instinto de trabalho eficiente". Este instinto surge na fase de selvageria pacífica (período pré-histórico) e indica a relação dos humanos com os materiais para a manutenção da vida em comunidade. Os indivíduos buscam instintivamente, motivados por hábitos de pensamento de uma sociedade, atividades úteis e eficazes como uma forma de alcançar seus objetivos, com repúdio à futilidade e valor à cooperação (Veblen, 1898).

Conforme Veblen (1898), a história cultural demonstra que os seres humanos desenvolvem atividades que resultam em bens ou serviços úteis. Não se deve generalizar que os indivíduos são, por natureza, esportistas, mas sim que possuem o "instinto de trabalho eficiente", uma vez que dispõem, no geral, de um comportamento cooperativo, não individualista. "What meets unreserved approval is such conduct as furthers human life on the whole, rather than such as furthers the invidious or predatory interest of one as against another" (Veblen, 1898, p. 192).

(11) A curiosidade vã desconsidera os elementos pecuniários (Monasterio, 1998).

(12) O "instinto de trabalho eficiente" e o "instinto predatório" possuem importância primordial pois são os pontos de partida para entendimento do pensamento vebleniano (Silva, 2010). 
Mesmo que Veblen (1898) admita que o ser humano possui, em certa medida, "aversão ao trabalho", fruto normalmente da sobrecarga de atividades, o autor é objetivo quando considera que as pessoas são principalmente impulsionadas pelo "instinto de trabalho eficiente". Para o autor, a "aversão ao trabalho" é um hábito da mente; o "instinto de trabalho eficiente" é, como o próprio nome sugere, um instinto, e representa uma característica necessária à sobrevivência humana. $\mathrm{O}$ "instinto de trabalho eficiente", assim como outros instintos básicos e essenciais, tem dominância para Veblen e é fundamental para garantir a manutenção da espécie.

De acordo com Veblen (1898), duas regras de conduta introduzidas pelo "instinto de trabalho eficiente" são: a eficiência e a serventia, valores que indicam o "conforto espiritual" de cada indivíduo perante a sociedade, pois "the degree of effectiveness with which he lives up to the accepted standard of efficiency in great measure determines his contentment with himself and his situation. A wide or persistent discrepancy in this respect is a source of abounding spiritual discomfort" (Veblen, 1898, p. 196).

Além de o instinto de realizar trabalhos úteis ser uma forma de garantia da vida da sociedade, os indivíduos agem desta maneira também para causar boa impressão aos demais, o que demonstra o caráter seletivo da vida em comunidade; os indivíduos estão em constantes processos de adaptação e aceitação (Veblen, 1898). Com o passar do tempo, as habilidades entre os indivíduos passam a ser comparadas e o reconhecimento e a estima tornam-se objetivos de realização, com maior espaço para as condições de emulação. "And what comes to be compared in an invidious comparison of this kind between agents is the force which the agent is able to put forth, rather than the serviceability of the agent's conduct"'(Veblen, 1898, p. 197).

O “instinto predatório", por sua vez, apresenta-se na passagem de uma fase de selvageria pacífica para uma etapa predatória, de modo que a competição entre os indivíduos se acirra e a força e a luta ganham caráter honorífico (Veblen, 1983).

The conditions favorable to the growth of a habit of emulative demonstration of force are (1) the frequent recurrence of conjunctures that call for a great and sudden strain, and (2) exposure of the individual to a large, and especially to a shifting, human environment whose approval is sought. These conditions are not effectually met on the lower levels of savagery, such as human culture must have been during the early days of the use of tools. Accordingly, relatively little of the emulative spirit is seen in communities that have retained the archaic, peaceable constitution, or that have reverted to it from a higher culture (Veblen, 1898, p. 198).

Apesar de conflitar com o "instinto de trabalho eficiente", o "instinto predatório" deriva deste e possibilitou o surgimento de diversas instituições, como: a propriedade privada, o status, o esporte, a classe ociosa (Silva, 2010). Como já 
apresentado em seção anterior, com o passar do tempo, o "instinto predatório" possibilitou o surgimento da classe ociosa e do comportamento de consumo conspícuo, por conta de sua ênfase na busca da superioridade e do poder pecuniário (Veblen, 1983).

Esta relação com o momento histórico também justifica a característica de hereditariedade dos instintos. Para Veblen (1914), os instintos possuem traços hereditários não devido às características fisiológicas do ser humano, como algo transmitido de forma intacta e imutável, mas sim às condições históricas de uma sociedade, uma vez que as pessoas migram para diferentes lugares, as raças e culturas se misturam e o ambiente se modifica.

With a change in the situation, therefore, such as may come with the migration of a given racial stock from one habitat to another, or with an equivalent shifting growth of culture or change of climate, the requirements of survival are likely to change. Indeed, so grave are the alterations that may in this way supervene in the current requirements for survival, that any given racial stock may dwindle and decay for no other reason than that the growth of its culture has come to subject the stock to methods of life widely different from those under which its type of man originated and made good its fitness to survive (Veblen, 1914, p. 16).

Esta ideia fortalece a importância de analisar conjuntamente os hábitos e os instintos, para a compreensão da mudança institucional.

[...] The habitual elements of human life change unremittingly and cumulatively, resulting in a continued proliferous growth of institutions. Changes in the institutional structure are continually taking place in response to the altered discipline of life under changing cultural conditions, but human nature remains specifically the same (Veblen, 1914, p. 18).

Ou seja, de acordo com Veblen (1914, p. 13), "the instincts are hereditary traits", e mesmo que seja identificada certa estabilidade com a transmissão de instintos entre as gerações, mudam as características habituais da vida humana e surgem outras instituições. "Changes in the institutional structure are continually taking place in response to the altered discipline of life under changing cultural conditions, but human nature remains specifically the same" (Veblen, 1914, p. 18).

Por mais que os instintos se verifiquem em diferentes etapas históricas, podem se manifestar de formas diferentes e seguir trajetórias diversas, de acordo com o quadro social e institucional vigentes. Conforme Veblen (1914), esses meios existentes para alcançar um fim envolvem heranças de hábitos de pensamento que pertenciam às antigas gerações.

A cognição dos indivíduos deve ser entendida como incorporada também em elementos sociais e institucionais (Nooteboom, 2007). As mensagens que são 
passadas pelas instituições irão depender da maneira como cada indivíduo, em particular e sob interferência do meio, absorve e interpreta as informações que envolvem o processo decisório.

Os hábitos compõem o processo cognitivo e incluem aspectos com maior ou menor nível de raciocínio: "some come about from an initial deliberation, others from instinctive reaction or emulation. Whatever its origin, a habit is normally a proclivity to act or think removed from the sphere of continuous deliberation" (Almeida; Pessali, 2011, p. 379). Os avanços nos estudos sobre comportamentos e indivíduos devem fazer referência aos aspectos cognitivos, como a racionalidade limitada, os processos cognitivos e as heurísticas, mas também aos aspectos institucionais, que incluem os hábitos e os instintos.

Os elementos cognitivos e institucionais estão presentes ao longo da evolução da espécie humana, e manifestam-se no comportamento muitas vezes sem que os indivíduos reflitam sobre isso. "[...] Quando nos "damos por gente" percebemos que o que está à nossa volta é a construção dos que nasceram antes e que tudo aquilo em alguma medida nos dá contornos cognitivos - o que são nossos hábitos, nossas razões, nossas aspirações, metas e insatisfações" (Pessali, 2015, p. 141).

Entretanto, sabendo que os hábitos e os instintos devem ser analisados de forma conjunta para formar os aspectos institucionais do comportamento, e que se aproximam dos aspectos cognitivos, pois ambos incluem processos do pensamento, resta agora encontrar um elemento que possa ser utilizado como conciliador das dimensões cognitivas e institucionais abordadas neste artigo.

\section{Considerações finais}

Este artigo apresentou os aspectos institucionais do comportamento dos indivíduos na Economia por meio da abordagem vebleniana. Com base na discussão sobre instintos e hábitos, percebeu-se que ambos elementos possuem ligação com a dimensão cognitiva e social do comportamento, o que amplia a visão tradicional de "homem econômico". A abordagem institucionalista vebleniana trouxe contribuições para o estudo do comportamento dos indivíduos na Economia.

Compreender o comportamento dos indivíduos em uma dimensão cognitiva e institucional vai além de descrever uma decisão ou escolha, ou seja, envolve a análise do processo que antecede a ação, como é o caso do comportamento de “consumo conspícuo". Nos fenômenos propostos por Veblen, o consumo dispõe de características que ultrapassam a utilidade física, constitui a formação da imagem pessoal perante um grupo. Com o consumo é possível demonstrar o poder pecuniário do indivíduo, e isto é resultado de instintos e hábitos. O "consumo conspícuo" e a mudança nas condições de emulação descrevem a complexidade do comportamento 
humano frente aos aspectos cognitivos e institucionais, sendo essenciais para compreender o "instinto de trabalho eficiente" e outros instintos veblenianos. Enquanto a maior parte dos instintos é mantida entre gerações, as mudanças nas instituições correspondem às mudanças nos hábitos de pensamento de uma sociedade, como seria, por exemplo, a moda - uma instituição ou um hábito de pensar - provocar a alteração no hábito de consumo de certos bens que configuram status.

Os instintos e os hábitos formam o "homem vebleniano" e indicam que a racionalidade pode ser encontrada em menor ou maior grau; a existência da razão não é questionada, mas sim a forma como ela é compreendida na Economia. $\mathrm{O}$ indivíduo visto aqui não é racional no sentido perfeito do "homem econômico", possui limitações cognitivas e comporta-se de acordo com os contextos históricos e com as interações sociais. Muitos comportamentos podem resultar do instinto que, apesar de ser inato à vida humana, possui características racionais, pois é preciso consciência para atingir um fim específico - no sentido vebleniano. Os hábitos, por sua vez, são formados na sociedade e por ela difundidos e possuem aspectos racionais no momento em que são criados e exercidos, mas, de outro lado, como os instintos, não exigem grande esforço da mente, já que representam atividades que os indivíduos estão acostumados a realizar.

O estudo do comportamento dos indivíduos envolve muito mais do que o apontamento de uma decisão ou do que a constatação de uma preferência. Por mais que a ação final compita ao indivíduo, a formação de seu pensamento, que resulta na escolha final, inclui hábitos e instintos, que compõem os aspectos institucionais e se relacionam com a cognição. As pessoas não são apenas individualizadas ou unicamente socializadas, possuem aspectos individuais e sociais.

Por fim, se a pretensão é analisar o comportamento humano, acredita-se que seria importante o estudo do que são, onde estão inseridos e quem são os indivíduos, e não apenas conjecturar sobre seus comportamentos e influências. Quando o comportamento e os indivíduos são analisados com base nos aspectos cognitivos e institucionais, entende-se que devem existir mais estudos que reforcem a dimensão ontológica na Economia, o que permite o aprofundamento deste artigo e a realização de novas pesquisas.

\section{Referências bibliográficas}

ALMEIDA, F.; PESSALI, H. Institutions, firms and consumers choice: extending neoschumpeterian competition to consumption. Evolutionary and Institucional Economics Review, v. 7, n. 2, p. 373-394, 2011.

CONCEIÇÃO, O. A. C. Instituições, crescimento e mudança na ótica institucionalista. Porto Alegre: FEE, 2002. (Teses FEE, n. 1). 
CONCEIÇÃO, O. A. C. Há compatibilidade entre a "tecnologia social" de Nelson e a "causalidade vebleniana" de Hodgson? Revista de Economia Política, v. 32, n. 1, p. 109-127, jan./mar., 2012.

COSGEL, M. M. Consumption institutions. Review of Social Economy, v. 55, n. 2, p. 153-171, 1997.

DALAL, M. N. Questioning consumerism. Journal of Economics and Development Studies. American Research Institute for Policy Development, New York, v. 2, n. 1, p. 1-29, Mar. 2014.

DOLFSMA, W. Mediated preferences - How institutions affect consumption. Journal of Economic Issues, v. XXXVI, n. 2, p. 449-457, Jun. 2002.

HODGSON, G. M. Economics and institutions: a manifesto for a modern institutional economics. Philadelphia: University of Pennsylvania Press, 1988. Chap. 1 and 6.

HODGSON, G. M. Institutional economics: surveying the 'old' and the 'new'. Metroeconomica, v. 44, n. 1, p. 1-28, 1993.

HODGSON, G. M. The Approach of Institutional Economics. Journal of Economic Literature, v. XXXVI, p. 166-192, Mar. 1998.

HODGSON, G. M. Choice, habit and evolution. Journal of Evolutionary Economics, n. 20 , p. $1-18,2010$.

HODGSON, G. M. The evolution of institutions: an agenda for future theoretical research. Constitutional Political Economy, v. 13, p. 111-127, 2002.

HODGSON, G. M. Toward an evolutionary and moral science: remarks on receiving the Veblen-commons award. Journal of Economic Issues, p. 1-13, Jan. 2012.

LUZ, M. R. S.; FRACALANZA, P. S. A gênese do indivíduo e o indivíduo da gênese: uma abordagem multidisciplinar acerca do papel da ideologia cristã nas origens do homem (econômico). Economia, Brasília, v. 14, n. 1A, p. 189-210, jan./abr., 2013.

MONASTERIO, L. M. Guia para Veblen: um estudo acerca da Economia Evolucionária. Pelotas: EDUFPEL, 1998. 128p.

NOOTEBOOM, B. Elements of a cognitive theory of the firm. Chap. 6. In: KRECKÉ, E.; KRECKÉ, C.; KOPPL, R. G. (Eds.). Cognition and economics. Oxford, Amsterdam: Elsevier, 2007.

PESSALI, H. F. Nanoelementos da mesoeconomia: uma economia que não está nos manuais. Curitiba: Ed. UFPR, 2015. 144p. 
RUTHERFORD, M. Institutions in economics: the old and the new institutionalism. Chap. 1. Cambridge: Cambridge University Press, 1994.

SILVA, V. L. da. Fundamentos do institucionalismo na teoria social de Thorstein Veblen. Política \& Sociedade, v. 9, n. 17, p. 289-323, out. 2010.

VEBLEN, T. The instinct of workmanship and the irksomeness of labor. American Journal of Sociology, v. 4, n. 2, p. 187-201, Sept. 1898.

VEBLEN, T. The limitations of marginal utility. Journal of Political Economy, v. 17, n. 9 , p. 620-636, Nov. 1909.

VEBLEN, T. The instinct of workmanship and the state of the industrial arts. New York: The Macmillan Company, 1914.

VEBLEN, T. The place of science in modern civilization. New York: B. W. Huebsch, 1919.

VEBLEN, T. [1899]. A teoria da classe ociosa: um estudo econômico das instituições. São Paulo: Abril Cultural, 1983.

VEBLEN, T. [1898]. Why is economics not an evolutionary science? Cambridge Journal of Economics, v. 22, p. 403-414, 1998. 